Original Paper http://ajol.info/index.php/ijbcs http://indexmedicus.afro.who.int

\title{
Etude ethnobotanique des plantes utilisées dans le traitement de la stérilité féminine dans les départements de l'Ouémé et du plateau au Sud Bénin
}

\author{
Victorin HOUMENOU ${ }^{1 *}$, Arlette ADJATIN ${ }^{2}$, Monique Gbèkponhami TOSSOU ${ }^{1}$, \\ Hounnakpon YEDOMONHAN ${ }^{1}$, Alexandre DANSI ${ }^{2}$, Joachim GBENOU ${ }^{3}$ et \\ Akpovi AKOEGNINOU ${ }^{1}$ \\ ${ }^{1}$ Laboratoire de Botanique et Écologie Végétale (LaBEV), Faculté des Sciences et Techniques (FAST), \\ Université d'Abomey-Calavi, Bénin, 01 BP 4521 Cotonou Bénin. \\ ${ }^{2}$ Laboratoire de Biotechnologie, des Ressources Génétiques et Amélioration des espèces Animales et \\ Végétales(BIORAVE), Université Nationale des Sciences, Technologies, Ingénieries et Mathématiques \\ d'Abomey (UNSTIM/Abomey) BP : 14, Dassa-Zoumè-Bénin. \\ ${ }^{3}$ Laboratoire de Pharmacognosie et des Huiles Essentielles, Faculté des Sciences et Techniques (FAST), \\ Université d'Abomey-Calavi, Bénin 01 BP 918 Cotonou-Bénin. \\ *Auteur correspondant, E-mail : moncredo2002@yahoo.fr; Tél : (+229) 97194830
}

\section{RESUME}

La procréation a été et continue d'être de nos jours la raison fondamentale du mariage en Afrique depuis les temps immémoriaux. L'infécondité du couple est vue comme une fatalité que ce dernier doit combattre à tout prix durant une bonne partie de sa vie. En vue de connaître les recettes utilisées au Bénin pour traiter la stérilité féminine, une enquête a été entreprise en direction des tradithérapeutes et des personnes ressources dans les départements de l'Ouémé et du Plateau au Sud-Bénin. Au total 93 tradithérapeutes et personnes ressources ont été enquêtés. Il s'agit d'une enquête ethnobotanique basée sur des interviews individuelles semi-structurées qui a permis de recenser 123 recettes dans lesquelles interviennent 90 espèces végétales appartenant à 86 genres et 47 familles dont les plus représentées sont les Leguminosae (10\%), les Euphorbiaceae (6,67\%), les Annonaceae (5,55\%). Les organes des espèces comme Anchomanes difformis, Aframomum melegueta, Allium cepa, Baphia nitida, Carissa spinarum, Elaeis guineensis, Garcinia kola, Kigelia africana, Monodora myristica, Morinda lucida, Musa sapientum, Olax subscorpioidea, Pupalia lappacea, Tetrapleura tetraptera, Piper guineense, Pterocarpus erinaceus, Xylopia aethiopica sont les plus cités dans les recettes. Ces plantes contribuent à soulager plusieurs couples infertiles et méritent d'être protégées pour la postérité.

(C) 2017 International Formulae Group. All rights reserved.

Mots clés : Infécondité, recettes, tradithérapeutes, personnes ressources, Sud-Bénin.

\section{The ethnobotanical study of plants used in women infertility treatment in the departments of Oueme and Plateau in South of Benin}

\section{ABSTRACT}

Procreation has been and continues to be today the fundamental reason for marriage in Africa since immemorial time. Infertility of the couple is seen as a fatality that it seeks to fight at all costs for a good part of his life. To know the recipes used in Benin to treat female infertility, a survey was undertaken towards the 
traditional healers and resource persons in the departments of Ouémé and Plateau in South of Benin Republic. On the whole, 93 traditional healers and resource persons were surveyed. This is an ethnobotanical sample survey which has permitted to identify the causes of female infertility and 123 recipes in which involved 90 plant species belonging to 86 genera and 47 families, the most represented being Leguminosae (10\%), Euphorbiaceae (6.67\%), Annonaceae (5.55\%). The organs of species such as Afromomum melegueta, Allium cepa, Baphia nitida, Carissa spinarum, Elaeis guineensis, Garcinia kola, Kigelia africana, Monodora myristica, Mrinda lucida, Musa sapientum, Olax subscorpioidea, Pupalia lappacea, Tetrapleura tetraptera, Pterocarpus erinaceus, Xylopia aethiopica are the most cited in the recipes. These plants contribute to relieve several infertile couples and deserve to be protected for posterity.

(C) 2017 International Formulae Group. All rights reserved.

Keywords: Infertility, recipes, traditional healers, resource persons, South of Benin.

\section{INTRODUCTION}

L'utilisation des plantes médicinales, $\mathrm{du}$ fait de leurs propriétés thérapeutiques, cosmétiques, chimiques, diététiques, pharmaceutiques, agro-alimentaires et industrielles est une pratique ancienne (Keirungi et Fabricius, 2005; Lahsissene et al., 2009). Selon CTA (2007) cité par Béné et al. (2016), les plantes médicinales font partie de l'histoire de tous les continents : en Chine et en Inde, à travers les siècles, le savoir concernant les plantes s'est organisé, documenté et a été transmis de génération en génération. Dans les pays industrialisés, la consommation des produits pharmaceutiques et les pratiques de phytochimie font augmenter sans cesse la demande en matières premières d'origine végétale. Dans les pays en voie de développement, cette demande croissante est liée principalement aux pratiques de médecine traditionnelle qui répondent, dans une large mesure, à la demande de soins des populations rurales (Betti et Lejoly, 2000). Actuellement, la médication par les plantes connaît un regain d'intérêt grâce aux études scientifiques basées sur les méthodes analytiques et les expérimentations (Lahsissene et al., 2009). Cependant, dans ces pays où une grande diversité des plantes médicinales existe, les recherches sur la connaissance et l'utilisation de celles-ci restent encore faibles ainsi que les échanges d'expériences et d'informations (Delvaux et Sinsin, 2002). La disparition des plantes médicinales et les connaissances endogènes qui leur sont associées, constituent une menace pour beaucoup de pays en voie de développement où les soins sanitaires dépendent beaucoup de ces ressources (Delvaux et Sinsin, 2002). Parmi les maladies traitées par la médecine traditionnelle, la stérilité féminine occupe une place de choix (Adomou, 2012). Dans la quasi-totalité des sociétés africaines, l'enfant occupe une place primordiale et devient de ce fait la raison de la survie ou non d'un couple; la femme stérile, privée du seul principe naturel pouvant la valoriser, se trouve diminuée (Akare Biyoghe, 2010). Ainsi, une femme sans enfant est considérée comme un déshonneur pour sa famille (Déléké Koko et al., 2009). La présente étude vise à inventorier et documenter les principales plantes médicinales utilisées dans le traitement de la stérilité féminine dans les départements de l'Ouémé et du Plateau au Sud-Est du Bénin.

\section{MATERIEL ET METHODES \\ Milieu d'étude}

L'étude a été réalisée dans les départements de l'Ouémé et du Plateau au Sud-Est du Bénin. Dans ces deux départements qui ont une frontière commune avec le Nigéria (Figure 1), le climat est subéquatorial à quatre saisons: 2 saisons pluvieuses et 2 saisons sèches (Akoègninou et al., 2004), la pluviométrie varie entre $900 \mathrm{~mm}$ et $1300 \mathrm{~mm}$ par an et le sol est majoritairement ferralitique (Adomou et al., 2007). Dans le département de l'Ouémé, 3 communes à savoir Adjohoun, Bonou et Dangbo ont été choisies sur les neuf que compte le département tandis que dans le département du plateau où il y a cinq 
communes, ce sont les communes d'AdjaOuèrè, Pobè et Kétou qui ont été retenues (Figure 1). Le choix de ces communes est dû au fait que ce sont des communes voisines et frontalières du Sud-Nigéria où la pluviométrie est élevée favorisant la biodiversité et où la médecine traditionnelle est développée. D'après le quatrième Recensement Général de la Population et de l'Habitation (RGPH42013), l'Ouémé et le Plateau comptent respectivement 1100404 habitants et 622372 habitants dominés essentiellement par les grands groupes ethniques Goun $(78,5 \%$ dans l'Ouémé) et Nagot (67,7\% dans le Plateau).

\section{Collecte des données}

Les données ont été collectées avec un questionnaire à travers une enquête ethnobotanique basée sur des interviews individuelles semi structurées (Saadou, 2008 ; Dansi et al., 2008; Dassou, 2015). Les informations collectées ont trait aux données géographiques (département, commune, village ou quartier de ville), à l'identité (nom, prénom, date et lieu de naissance, langues parlées) et expériences de l'enquêté, les causes de la stérilité féminine, les espèces utilisées, le mode de préparation (décoction, macération, trituration, etc.) et la composition de la recette ainsi que son mode d'utilisation.

Dix villages (Démè et Adjohoun dans la commune d'Adjohoun; Bonou et Tovo dans la commune de Bonou; Ké dans la commune de Dangbo ; Massè et Adja-Ouèrè dans la commune d'Adja-Ouèrè ; Issaba et Pobè dans la commune de Pobè ; Adakplamè dans la commune de Kétou) ont été sélectionnés au hasard dans la zone d'étude. Dans chaque village les personnes enquêtées sont des tradithérapeutes et personnes ressources reconnues par la société comme ayant une bonne connaissance des plantes médicinales. Plusieurs enquêtés étant analphabètes, un interprète a été recruté dans chaque village pour faciliter les échanges en langue du milieu.

Les espèces citées ont été à chaque fois photographiées puis échantillonnées et des herbiers ont été réalisés pour l'identification taxonomique (nom scientifique, famille) avec la Flore analytique du Bénin (Akoègninou et al., 2006) et autres documents de référence (Adjanohoun et al., 1989) disponibles à l'Herbier National du Bénin.

\section{Analyse statistique des données}

Les données obtenues ont été analysées avec le logiciel R. Le test du khi-deux (test de conformité à une distribution uniforme) a été réalisé pour évaluer la perception par les populations de la prépondérance relative des différentes causes incriminées dans la stérilité féminine, des différents types morphologiques des espèces de plantes utilisées (arbre, arbuste, herbe ou liane), du nombre de recettes suivant les causes, des différents types d'organes de plantes et des divers modes de préparation des recettes utilisés.

Pour catégoriser les enquêtés, les classes d'âges proposées par Assogbadjo et al. (2008) ont été utilisées et se présentent comme suit: les jeunes (23 à 29 ans), les adultes (30 à 59 ans) et les anciens (60 à 72 ans).

Pour évaluer l'importance socioculturelle des plantes utilisées dans le traitement de la stérilité féminine, plusieurs paramètres ont été calculés :

- Indice de diversité (ID) des enquêtés. La valeur de diversité indique la diversité des catégories d'usage de l'espèce et présente comment cette connaissance est distribuée parmi les enquêtés (Byg et Baslev, 2011). L'indice de diversité permet une comparaison de la diversité des usages de la pharmacopée suivant les différentes modalités formant ces groupes au sein de l'échantillon. Indice de diversité $\mathrm{ID}=\mathrm{Ux} / \mathrm{Ut}$ avec $\mathrm{Ux}$ : nombre d'utilisations citées par un enquêté et Ut: nombre total d'utilisations cités par les enquêtés; cet indice mesure le nombre d'enquêtés qui utilisent l'espèce et la manière dont cette connaissance est distribuée entre les enquêtés.

- Indice d'équitabilité des enquêtés
permet une comparaison du niveau
d'homogénéité des connaissances sur les
usages de la pharmacopée en fonction des
modalités composant les groupes socio-
économiques. Indice d'équitabilité IE =


ID/IDmax avec ID : valeur de la diversité des enquêtés, IDmax: valeur maximale de la diversité obtenue chez les enquêtés. Cet indice mesure le degré d'homogénéité de connaissances des enquêtés.

- Valeurs consensuelles sur les types d'usage $\mathrm{CTU}=(\mathrm{TU} / \mathrm{Ut}) / \mathrm{S}$ avec $\mathrm{TU}:$ le nombre de fois que l'usage est rapporté, Ut : le nombre total d'usages et $\mathrm{S}$ : types d'usages classés par catégorie. Elle mesure le degré de concordance entre les enquêtés au regard des usages faits de l'espèce.

- Fréquence relative de citation (FRC) donnée par la formule utilisée par Dossou et al., (2012); $\mathrm{FRC}=\mathrm{S} / \mathrm{N}$ x 100 où $\mathrm{S}$ est le nombre de personnes ayant fourni une réponse par rapport à une utilisation donnée et $\mathrm{N}$ est le nombre total de personnes interviewées. ontribution de chaque plante dans la constitution des recettes $(\mathrm{Cpr})$ donnée par la formule utilisée par Dassou et al., (2014) : $\mathrm{Cpr}=\mathrm{Nr} \times 100 / \mathrm{Nt}$ où $\mathrm{Nr}$ représente le nombre de recettes sollicitant la plante et $\mathrm{Nt}$ le nombre total de recettes. Elle permet de connaître la fréquence d'implication d'une plante dans les recettes.

- Valeur d'usage (VUe) par espèce : le nombre de fois que l'espèce est citée dans les recettes. Par conséquent, cette valeur est toujours un nombre entier contrairement aux autres indices.

- Valeur d'importance (VI) : c'est le rapport de la valeur d'usage par espèce (VUe) par le nombre d'espèces $(\mathrm{N})$. VI $=$ Vue $/ \mathrm{N}$ avec $\mathrm{N}$, le nombre d'espèces.

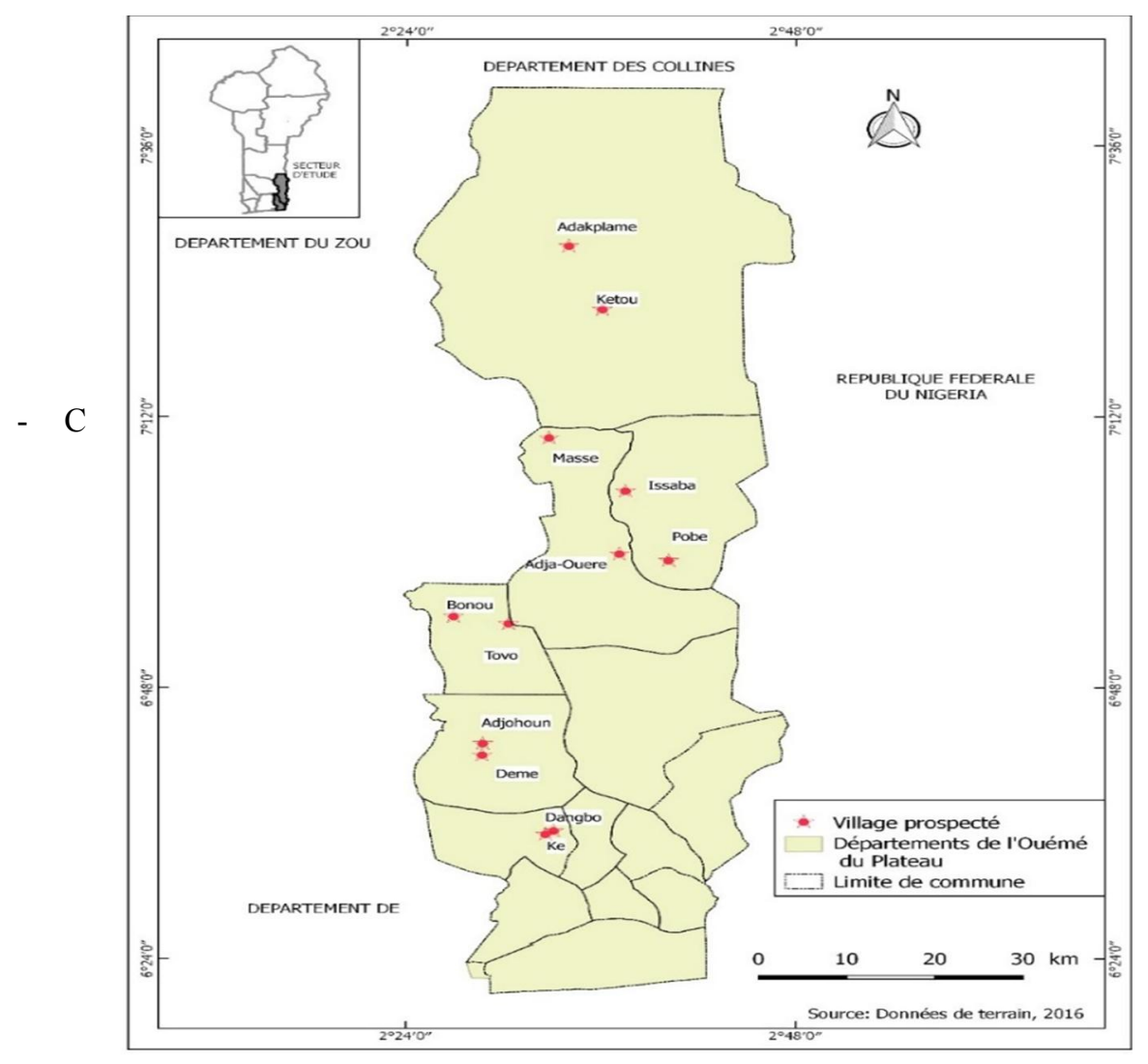

Figure 1 : Carte des départements de l'Ouémé et du Plateau. 
RESULTATS

Caractéristiques socio-économiques des enquêtés

Les enquêtes réalisées au Sud-Bénin ont permis de recueillir des informations sur le traitement contre la stérilité féminine. Tous les enquêtés sont de sexe masculin $(100 \%)$ car toutes les personnes du sexe féminin rencontrées se sont désistées au profit de leur conjoint. En se basant sur l'âge des enquêtés et des classes prédéfinies, seulement $11,83 \%$ sont des jeunes (classe de 23 à 29 ans) tandis que $70,97 \%$ sont des adultes (classe de 30 à 59 ans) et $17,20 \%$ sont des anciens (classe de 60 à 72 ans). Avec un risque bien inférieur à un $1 / 1000000$, la classe adulte est beaucoup plus représentée que les autres $(\mathrm{X}$-squared $=$ 59.6774, $\mathrm{df}=2$, p-value $=1.1 \mathrm{e}-13)$. De plus, elle est plus forte que l'ensemble formé par les deux autres classes (X-squared $=16.3548$, $\mathrm{df}=1, \mathrm{p}$-value $=5.252 \mathrm{e}-05)$. Avec un risque bien inférieur à un $1 / 1000000$, le groupe des gouns $(39,78 \%)$ a l'effectif le plus élevé (Xsquared $=55.4409, \mathrm{df}=4, \mathrm{p}$-value $=2.626 \mathrm{e}$ 11) par rapport aux groupes Yoruba $(32,25 \%)$ et Mahi (27,95\%). Cependant, il y a une équitabilité entre les 3 groupes $(\mathrm{X}$-squared $=$ $3.1613, \mathrm{df}=2, \mathrm{p}$-value $=0.2058)$. Trois niveaux d'instruction sont plus représentés que les autres; il s'agit des analphabètes $(35,48 \%)$, le niveau primaire $(27,95 \%)$ et le premier cycle des collèges $(30,10 \%)$; les enquêtés du second cycle et étudiants sont en nombre inférieur aux premiers $(\mathrm{X}$-squared $=$ $26.4086, \quad \mathrm{df}=4, \mathrm{p}$-value $=2.617 \mathrm{e}-05)$ (Tableau 1).

Analyse des valeurs ethnobotaniques de la pharmacopée dans le traitement de la stérilité féminine

L'indice de diversité a une distribution conforme à celle de la loi normale de moyenne 0,01 et d'écart type 0,00 (Tableau 2). L'indice d'équitabilité a une distribution significativement différente de celle de la loi normale dont la moyenne est de 0,32. Le coefficient de variation de l'indice d'équitabilité est relativement élevé $(49,23 \%)$; ce qui témoigne d'une grande dispersion relative des valeurs. Ainsi, les connaissances sur les usages de la pharmacopée dans le traitement des affections liées à la stérilité féminine ne sont pas équitablement réparties au sein des enquêtés par conséquent certains enquêtés ont plus de connaissances que d'autres (Tableau 2).

\section{Diversité des espèces végétales utilisées dans le traitement de la stérilité féminine}

$\mathrm{Au}$ total, 90 espèces intervenant dans 123 recettes dont $50,41 \%$ sont monospécifiques composées d'une seule plante ont été recensées (Tableau 3). Ces espèces se répartissent en 86 genres et 47 familles dont les plus représentées sont les Leguminosae (10\%), les Euphorbiaceae $(6,67 \%)$, les Annonaceae (5,55\%), les Rubiaceae (4,44\%) et les Apocynaceae $(3,33 \%)$. Les principales espèces végétales appartiennent au spectre des principaux types morphologiques que sont les arbres qui ont une proportion élevée (37\%) suivi des herbes, arbustes et lianes représentés respectivement par $35 \%, 17 \%$, et $11 \%$ (Figure 2). Plusieurs organes végétaux tels que les feuilles, les racines, les fruits, les écorces, les graines, les tubercules, les organes floraux et le faux tronc interviennent diversement dans la préparation des recettes utilisées contre la stérilité féminine. Les feuilles, les racines, les fruits et les écorces sont relativement plus cités que s'il y avait la perception d'une prépondérance équitable d'intervention, tandis que les graines, les tubercules, les plantes entières, les organes floraux et le faux tronc sont relativement moins cités que s'il y avait la perception d'une prépondérance équitable. De façon plus précise, les fréquences des feuilles, racines, fruits, écorces sont respectivement $23 \%, 20 \%, 19 \%, 15 \%$ alors que les graines, tubercules, plantes entières, organes floraux et faux tronc sont respectivement $7 \%, 6 \%, 5 \%$, 
$4 \%$ et $1 \%$ du nombre total des organes recensés (Figure 3 ).

\section{Diversité des usages suivant les caractéristiques socioéconomiques des enquêtés}

L'analyse de la variance effectuée sur les valeurs d'usage indiquent que la classe d'âge (P-value $>0,1>\alpha=0,05$ ), le niveau d'instruction (P-value $>0,1>\alpha=0,05)$ et la catégorie socioprofessionnelle (P-value $>0,1$ $>\alpha=0,05)$ de l'enquêté n'ont aucune influence sur les types d'usages qu'un enquêté fait des plantes dans le traitement des causes liées à la stérilité féminine contrairement aux catégories sociolingustiques (P-value $<0,001<\alpha=0,05$ ). Le groupe sociolinguistique Mahi présente plus d'informations sur l'usage de la pharmacopée dans le traitement des causes liées à la stérilité féminine que les groupes sociolinguistiques Yoruba et Goun qui ont des niveaux de connaissances similaires sur l'utilisation de la pharmacopée dans le traitement de ces affections.

\section{Valeurs consensuelles d'usage de la pharmacopée}

Pour l'ensemble des enquêtés, il se dégage des niveaux de consensus plus larges respectivement par ordre décroissant pour le traitement des affections telles que l'infertilité $(\mathrm{CTU}=0,04)$, le myome $(\mathrm{CTU}=0,03)$, les menstrues malodorantes $(\mathrm{CTU}=0.02)$ et le rejet de sperme $(\mathrm{CTU}=0,02)$. Le traitement de l'hyperthermie abdominale (CTU $=0.01)$ recueille un niveau de consensus beaucoup moins important que celui relatif à l'infertilité, au myome, aux menstrues malodorantes et au rejet de sperme. Les traitements des trompes bouchées $(\mathrm{CTU}=0.00)$ ainsi que ceux des autres affections réunies $(\mathrm{CTU}=0.01)$ sont crédités de niveaux de consensus plus faibles 10 fois moins importants que les précédents. Par ailleurs, l'influence de l'appartenance à un groupe sociolinguistique sur les niveaux de consensus révèle quelques particularités
(Figure 4). Les niveaux de consensus des Gouns et des Mahi sont les plus proches ; en effet par ordre décroissant des niveaux de consensus, les Mahi s'accordent plus pour le traitement de «l'infertilité » $(\mathrm{CTU}=0,04)$, des « myomes » $(\mathrm{CTU}=0,04)$ ainsi que des « menstrues malodorantes » $(\mathrm{CTU}=0,03)$; de même, les Goun et apparentés affichent un consensus plus élevé pour «l'infertilité " $(\mathrm{CTU}=0,04)$, les « myomes » $(\mathrm{CTU}=0,03)$ et les menstrues malodorantes $(\mathrm{CTU}=0,02)$. Les niveaux de consensus les plus éloignés entre les Mahi et les Gouns sont observées au niveau du traitement de l'hyperthermie pour lequel le niveau de consensus des usages de la pharmacopée par les Mahi $(\mathrm{CTU}=0,01)$ vaut 1,6 fois celui affiché par les Gouns et apparentés (CTU $=0,01)$. Quant aux Yoruba et apparentés, par ordre décroissant des niveaux de consensus, ils s'accordent beaucoup plus, en premier lieu de façon égale sur le traitement des «rejets de sperme» (CTU $=0,03$ ) et de l'infertilité (CTU=0,03), dans un second temps sur celui des «menstrues malodorantes » $(\mathrm{CTU}=0,03)$, dans un troisième temps sur celui des myomes. Ce groupe sociolinguistique se distingue par le niveau de consensus le plus élevé pour le traitement des «trompes bouchées » $(\mathrm{CTU}=0,01)$ et des «autres affections » $(\mathrm{CTU}=0,01)$. Le niveau de consensus des yoruba pour le traitement des «trompes bouchées » vaut au moins 1,8 fois celui des deux autres groupes sociolinguistiques et 5 fois celui des Mahi.

Importance relative des recettes par cause dans le traitement de la stérilité féminine

$\mathrm{Au}$ total, 13 causes responsables de la stérilité féminine sont recensées suivant la perception des enquêtés (Figure 5). D'une cause à une autre, le nombre de citations des recettes varie. Ainsi le nombre de recettes pour le traitement de chacune des causes de stérilité a une prépondérance qui varie d'une 
cause à une autre $(\mathrm{X}$-squared $=66.18, \mathrm{df}=7$, $\mathrm{p}$-value $=8.71 \mathrm{e}-12)$. Par conséquent, le nombre de recettes contre le myome, le rejet de sperme, les menstrues malodorantes, l'infertilité ou l'hyperthermie abdominale est relativement plus élevé que l'ensemble des recettes contre les trompes bouchées, la glaire de mauvaise qualité et autres causes. Parmi les 123 recettes recueillies auprès des populations, les proportions de chacune des affections se présentent comme suit: le myome $(24 \%)$, le rejet de sperme $(18 \%)$, les menstrues malodorantes (17\%), l'infertilité $(16 \%)$ et l'hyperthermie abdominale (14\%). Les trompes bouchées, avortement spontané, aménorrhée, glaire de mauvaise qualité, métrorragie sur grossesse, incompatibilité sanguine, règles douloureuses et l'utérus sale se partagent $11 \%$ des recettes.

\section{Mode de préparation et formes d'administration des recettes}

Les recettes utilisées dans le traitement de la stérilité féminine sont confectionnées suivant cinq (5) modes de préparation dont les fréquences de citations diffèrent significativement; par conséquent les modes de préparation ne sont pas équitablement cités $(\mathrm{X}$-squared $=35.12, \mathrm{df}=4, \mathrm{p}$-value $=4.389 \mathrm{e}$ 07). La décoction est le mode de préparation le plus utilisé avec $40 \%$ suivi de la macération qui représente 20\% (Figure 6). Le pilage, la trituration et la calcination sont également des modes de préparations représentant respectivement $15 \%, 13 \%$ et $12 \%$ des réponses.

Les formes d'administration dérivent des modes de préparation des recettes. C'est ainsi, que les décoctés, les macérés, les poudres et le triturât sont tous principalement administrés par voie orale. Mais les macérés et les décoctés sont utilisés à la fois par voie orale $(97 \%)$ et pour les toilettes intimes de la femme $(3 \%)$ alors que les poudres sont parfois utilisées pour fabriquer des pommades $(1 \%)$ et des ovules administrés dans le vagin.

L'étude de la spécificité des plantes à la confection des recettes a montré que $55,55 \%$ des plantes utilisées dans le traitement de la stérilité féminine interviennent dans la confection des recettes contre l'infertilité. Parmi ces plantes, 16 sont les plus représentées et citées au moins 2 fois par les enquêtés (Figure 7-a). Il ressort que Cnestis ferruginea, Xylopia aethiopica, Aframomum melegueta, Baphia nitida, Kigelia africana, Allium cepa, Olax subscorpioidea sont beaucoup plus indiquées dans le traitement de l'infertilité. Pour le myome, l'étude de spécificité a montré que 35,55\% des plantes recensées sont utilisées. La Figure 7-b présente les plantes les plus citées. Il s'agit de Garcinia kola, Musa sapientum, Nicotiana tabacum, Tetrapleura tetraptera, Xylopia aethiopica qui sont les plus indiquées dans le traitement du myome. Parmi les plantes recensées, 23,33\% sont utilisées dans la confection de recettes contre le rejet de sperme. Pupalia lappacea est l'espèce la plus utilisée suivie d'Aframomum melegueta, de Musa sapientum et de Cissampelos owariensis (Figure 7-c). Quant aux menstrues malodorantes, $20 \%$ des plantes recensées sont utilisées pour la confection de recettes. Parmi celles-ci, Baphia nitida, Pterocarpus erinaceus, Kigelia africana, Parkia biglobosa sont les plus citées. (Figure 7-d). Très peu de plantes sont utilisées de façon spécifique dans le traitement de l'hyperthermie abdominale. 
Tableau 1 : Caractéristiques socio-économiques des enquêtés.

\begin{tabular}{|c|c|c|}
\hline Paramètres & Catégories socioprofessionnelles & Proportions (\%) \\
\hline \multirow{3}{*}{ Classe d'âges } & - Jeunes $[16 ; 29]$ & $-11,83$ \\
\hline & - Adultes $[30 ; 59]$ & $-70,96$ \\
\hline & - Anciens [60; [ & $-17,20$ \\
\hline \multirow{3}{*}{ Groupes ethniques } & - Mahi & $-27,95$ \\
\hline & - Yoruba et apparentés & $-32,25$ \\
\hline & - Gouns et apparentés & $-39,78$ \\
\hline \multirow{5}{*}{ Niveaux d'instruction } & - Analphabètes & $-27,95$ \\
\hline & - Primaires & $-27,95$ \\
\hline & $-1^{\mathrm{er}}$ cycle & $-30,10$ \\
\hline & $-2^{\text {nd }}$ cycle & $-6,45$ \\
\hline & -Université & $-7,53$ \\
\hline \multirow{8}{*}{$\begin{array}{l}\text { Catégories } \\
\text { professionnelles }\end{array}$} & - Etudiants & $-2,15$ \\
\hline & - Salariés & $-12,90$ \\
\hline & - Entrepreneurs & $-16,12$ \\
\hline & - Retraités & $-1,075$ \\
\hline & - Agriculteurs & $-35,48$ \\
\hline & - Commerçants & $-4,30$ \\
\hline & - Tradithérapeutes & $-13,97$ \\
\hline & - Conducteurs de taxi-moto & $-13,97$ \\
\hline
\end{tabular}

Tableau 2: Indice de diversité et d'équitabilité des enquêtés.

\begin{tabular}{|c|c|c|c|}
\hline $\begin{array}{l}\text { Groupes socio- } \\
\text { économiques }\end{array}$ & Variables mesurées & $\begin{array}{l}\text { Indice de } \\
\text { diversité }(95 \%)\end{array}$ & $\begin{array}{l}\text { Indice } \\
\text { d'équitabilité } \\
(95 \%)\end{array}$ \\
\hline \multirow{3}{*}{ Catégories d'âges } & Jeune & $0,01 \pm 0,00$ & $0,31 \pm 0,10$ \\
\hline & Adulte & $0,01 \pm 0,00$ & $0,31 \pm 0,04$ \\
\hline & Ancien & $0,01 \pm 0,00$ & $0,35 \pm 0,07$ \\
\hline \multirow{3}{*}{$\begin{array}{l}\text { Groupes socio- } \\
\text { linguistiques }\end{array}$} & Goun et apparentés & $0,01 \pm 0,00$ & $0,27 \pm 0,04$ \\
\hline & Mahi & $0,01 \pm 0,00$ & $0,41 \pm 0,07$ \\
\hline & Yoruba et apparentés & $0,01 \pm 0,00$ & $0,30 \pm 0,05$ \\
\hline \multirow{4}{*}{ Niveaux d'instruction } & Illettré & $0,01 \pm 0,00$ & $0,31 \pm 0,05$ \\
\hline & Primaire & $0,01 \pm 0,00$ & $0,35 \pm 0,06$ \\
\hline & Secondaire & $0,01 \pm 0,00$ & $0,29 \pm 0,06$ \\
\hline & Universitaire & $0,01 \pm 0,00$ & $0,34 \pm 0,14$ \\
\hline \multirow{5}{*}{$\begin{array}{l}\text { Catégories } \\
\text { socioprofessionnelles }\end{array}$} & non actifs & $0,01 \pm 0,00$ & $0,20 \pm 0,00$ \\
\hline & Agriculteur & $0,01 \pm 0,00$ & $0,32 \pm 0,05$ \\
\hline & Traditherapeute & $0,01 \pm 0,00$ & $0,34 \pm 0,09$ \\
\hline & Actif non salarié & $0,01 \pm 0,00$ & $0,29 \pm 0,04$ \\
\hline & Salarié & $0,01 \pm 0,00$ & $0,42 \pm 0,18$ \\
\hline
\end{tabular}




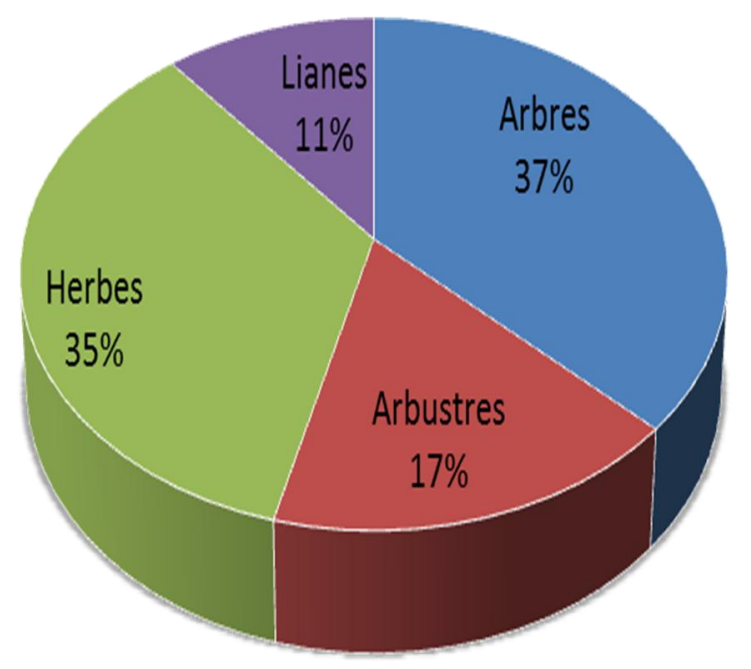

Figure 2 : Spectre des types morphologiques.

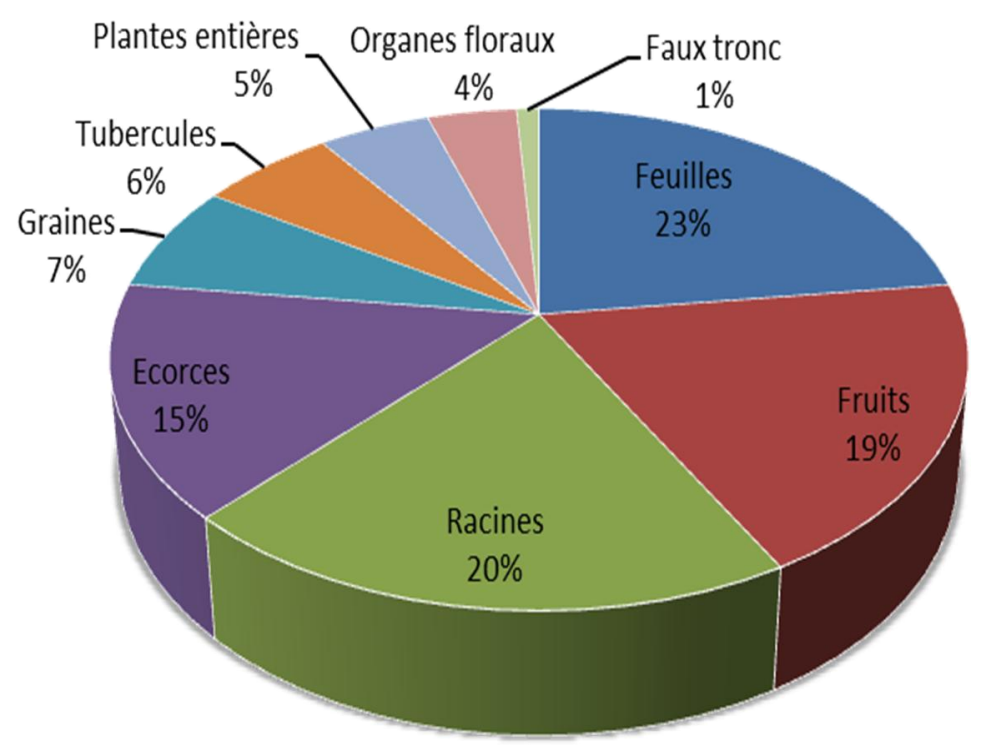

Figure 3 : Diagramme des différentes parties des plantes utilisées. 
V. HOUMENOU et al. / Int. J. Biol. Chem. Sci. 11(4): 1851-1871, 2017

Tableau 3 : Liste des espèces végétales citées dans les recettes et leurs caractéristiques.

\begin{tabular}{|c|c|c|c|c|c|c|c|c|c|c|c|}
\hline $\mathbf{N}^{\circ}$ & Noms scientifiques & Familles & TM & CPR & Organes & VUe & Mp & $\mathbf{V a}$ & MT & VI & VUoe \\
\hline 1 & Allium сера & Alliaceae & Herbe & 3,25 & Bulbe & 04 & $\begin{array}{l}\text { Déc, Déc } \\
\text { Mac, Déc }\end{array}$ & Orale & $\mathrm{M}, \mathrm{Ha}, \mathrm{I}$ & 0,04 & 1 \\
\hline 2 & Allium sativum & Alliaceae & Herbe & 0,81 & Bulbe & 01 & Déc & Orale & I & 0,01 & 1 \\
\hline 3 & Alternanthera pungens & Amaranthaceae & Herbe & 1,63 & Feuille & 02 & Pil, Déc, & Orale & $\mathrm{Rs}, \mathrm{Mm}$ & 0,02 & 1 \\
\hline 4 & Amaranthus spinosus & Amaranthaceae & Herbe & 0,81 & Racine & 01 & Pil & Voie vaginale & I & 0,01 & 1 \\
\hline 5 & Pupalia lappacea & Amaranthaceae & Herbe & 6,50 & $\begin{array}{l}\text { Feuille (2), Fruit } \\
\text { (6) }\end{array}$ & 8 & $\begin{array}{l}\text { Trit (1), Pil } \\
(3), \text { Calc (3) }\end{array}$ & Orale & Rs, Ha & 0,09 & $\begin{array}{l}0,25 \\
0,75\end{array}$ \\
\hline 6 & Spondias mombin & Anacardiaceae & Arbre & 2,44 & $\begin{array}{l}\text { Ecorce }(2) \\
\text { Feuille(1) }\end{array}$ & 3 & Déc & Orale & $\mathrm{M}, \mathrm{Mm}, \mathrm{I}$ & 0,03 & $\begin{array}{l}0,67 \\
0,33\end{array}$ \\
\hline 7 & Annona senegalensis & Annonaceae & Arbustre & 1,63 & Feuille & 02 & Pil & Orale & I & 0,02 & 1 \\
\hline 8 & Cleistopholis patens & Annonaceae & Arbre & 0,81 & Ecorce & 01 & Déc & $\begin{array}{l}\text { Orale, } \\
\text { Toilette intime }\end{array}$ & M & 0,01 & 1 \\
\hline 9 & Monodora myristica & Annonaceae & Arbre & 2,44 & Graine & 03 & $\begin{array}{l}\text { Déc (2) } \\
\text { Mac (1) }\end{array}$ & $\begin{array}{l}\text { Orale, } \\
\text { Toilette intime }\end{array}$ & M,I & 0,03 & 1 \\
\hline 10 & Uvaria chamae & Annonaceae & Liane & 0,81 & Racine & 01 & Déc & Orale & I & 0,01 & 1 \\
\hline 11 & Xylopia aethiopica & Annonaceae & Arbre & 6,50 & Fruit & 08 & $\begin{array}{l}\text { Déc (5) Mac } \\
(2), \text { Cal (1) }\end{array}$ & $\begin{array}{l}\text { Orale, } \\
\text { Toilette intime }\end{array}$ & M, I & 0,09 & 1 \\
\hline 12 & Carissa spinarum & Apocynaceae & Arbuste & 3,25 & Racine & 04 & Mac & $\begin{array}{l}\text { Orale, } \\
\text { Toilette intime }\end{array}$ & Rs, M & 0,04 & 1 \\
\hline
\end{tabular}


V. HOUMENOU et al. / Int. J. Biol. Chem. Sci. 11(4): 1851-1871, 2017

\begin{tabular}{|c|c|c|c|c|c|c|c|c|c|c|c|}
\hline 13 & Holarrhena floribunda & Apocynaceae & Arbre & 0,81 & Feuille & 01 & Trit & Orale & As & 0,01 & 1 \\
\hline 14 & Rauvolfia vomitoria & Apocynaceae & Arbuste & 2,44 & $\begin{array}{l}\text { Feuille } \\
\text { Ecorce } \\
\text { Racine }\end{array}$ & 03 & $\begin{array}{l}\text { Déc, } \\
\text { Mac }\end{array}$ & Orale & M & 0,03 & $\begin{array}{l}0,33 \\
0,33 \\
0,33\end{array}$ \\
\hline 15 & Anchomanes difformis & Araceae & Herbe & 3,25 & Tubercule & 04 & $\begin{array}{l}\text { Déc (2), Mac } \\
\text { (1), Pil (1) }\end{array}$ & Orale & I, Rs, Ha & 0,04 & 1 \\
\hline 16 & Elaeis guineensis & Arecaceae & Arbre & 3,25 & Spadice mâle & 04 & $\begin{array}{l}\text { Déc (4), Cal } \\
(3)\end{array}$ & Orale & $\mathrm{M}, \mathrm{Rd}$ & 0,04 & 1 \\
\hline 17 & Calotropis procera & Asclepiadaceae & Arbuste & 0,81 & Feuille & 01 & Pil & Orale & Rs & 0,01 & 1 \\
\hline 18 & $\begin{array}{l}\text { Acanthospermum } \\
\text { hispidum }\end{array}$ & Asteraceae & Herbe & 0,81 & Feuille & 01 & Déc & Orale & I & 0,01 & 1 \\
\hline 19 & Vernonia cinerea & Asteraceae & Herbe & 0,81 & Feuille & 01 & Déc & Orale & I & 0,01 & 1 \\
\hline $\mathbf{N}^{\circ}$ & Noms scientifiques & Familles & TM & CPR & Organes & VUe & Мp & $\mathbf{V a}$ & MT & VI & VUoe \\
\hline 20 & Kigelia africana & Bignoniaceae & Arbre & 8,13 & $\begin{array}{l}\text { Fruit (2), } \\
\text { Ecorce }(8)\end{array}$ & 10 & $\begin{array}{l}\text { Déc (7) Cal } \\
\text { (1) }\end{array}$ & Orale & $\begin{array}{l}\mathrm{Ha}, \mathrm{Mm}, \mathrm{I}, \\
\mathrm{M}, \mathrm{Rd}\end{array}$ & 0,11 & $\begin{array}{l}0,2 \\
0,8\end{array}$ \\
\hline 21 & Newbouldia laevis & Bignoniaceae & Arbre & 1,63 & Feuille & 02 & Déc, Trit & Orale & I, Ha & 0,02 & 1 \\
\hline 22 & Heliotropium indicum & Boraginaceae & Herbe & 0,81 & $\begin{array}{l}\text { Plante } \\
\text { Entière }\end{array}$ & 01 & Trit & Orale & I & 0,01 & 1 \\
\hline 23 & Crateva adansonii & Capparaceae & Arbre & 0,81 & Feuille & 01 & Déc & Orale & M & 0,01 & 1 \\
\hline 24 & Carica papaya (mâle) & Caricaceae & Arbre & 2,44 & Racine & 03 & Cal, Pil, Mac & Orale & M & 0,03 & 1 \\
\hline 25 & $\begin{array}{l}\text { Chenopodium } \\
\text { ambrosioides }\end{array}$ & Chenopodiaceae & Herbe & 0,81 & Feuilles & 01 & Trit & Orale & M & 0,01 & 1 \\
\hline 26 & Garcinia kola & Clusiaceae & Arbre & 4,88 & Graine & 06 & $\begin{array}{l}\text { Pil (3) } \\
\text { Mac (2) } \\
\operatorname{Déc}(1)\end{array}$ & Orale & $\begin{array}{l}\text { M, I, Ha, } \\
\text { Rs, }\end{array}$ & 0,07 & 1 \\
\hline
\end{tabular}


V. HOUMENOU et al. / Int. J. Biol. Chem. Sci. 11(4): 1851-1871, 2017

\begin{tabular}{|c|c|c|c|c|c|c|c|c|c|c|c|}
\hline \multirow[t]{2}{*}{27} & Combretum racemosum & Combretaceae & Liane & 1,63 & Feuille & 02 & Déc & Orale & & & \\
\hline & & & & & & & & & $\mathrm{I}, \mathrm{Tb}$ & 0,02 & 1 \\
\hline \multirow[t]{2}{*}{28} & Cnestis ferruginea & Connaraceae & Arbuste & 4,06 & Feuille (3) & 05 & Cal (2), Déc & Orale & $\mathrm{Mm}, \mathrm{I}$ & 0,05 & 0,6 \\
\hline & & & & & Racine(2) & & (2), Pil (1) & & & & 0,4 \\
\hline \multirow[t]{2}{*}{29} & Rourea coccinea & Connaraceae & Liane & 4,06 & Feuille (3) & 05 & Trit (3) & Orale & $\mathrm{M}, \mathrm{Mm}$ & 0,05 & 0,6 \\
\hline & & & & & Racine(2) & & Déc (2) & & Ha, Rs, I & & 0,4 \\
\hline 30 & Bryophyllum pinnatum & Crassulaceae & Herbe & 0,81 & Feuille & 01 & Déc & Orale & Gmq & 0,01 & 1 \\
\hline 31 & Citrullus colocynthis & Cucurbitaceae & Herbe & 0,81 & Fruit & 01 & Déc & Orale & $\mathrm{Mm}$ & 0,01 & 1 \\
\hline 32 & Momordia charantia & Cucurbitaceae & Liane & 0,81 & Racine & 01 & Déc & Orale & I & 0,01 & 1 \\
\hline 33 & Dracaena arborea & Dracaenaceae & Arbuste & 0,81 & Racine & 01 & Mac & Toilette intime & $\mathrm{M}$ & 0,01 & 1 \\
\hline 34 & Sansevieria liberica & Dracaenaceae & Herbe & 0,81 & Racine & 01 & Mac & Orale & Rs & 0,01 & 1 \\
\hline 35 & Acalypha ciliata & Euphorbiaceae & Herbe & 0,81 & Feuille & 01 & Déc & Orale & I & 0,01 & 1 \\
\hline 36 & Bridelia ferrugina & Euphorbiaceae & Arbuste & 0,81 & Ecorce & 01 & Déc & Orale & I & 0,01 & 1 \\
\hline 37 & Croton zambesicus & Euphorbiaceae & Arbre & 0,81 & Feuille & 01 & Pil & Orale & I & 0,01 & 1 \\
\hline 38 & Jatropha curcas & Euphorbiaceae & Arbuste & 1,63 & Racine & 02 & Mac & Orale & Ha, M & 0,02 & 1 \\
\hline \multirow[t]{2}{*}{39} & Jatropha gossypiifolia & Euphorbiaceae & Arbuste & 2,44 & Feuille (2) & 03 & Trit (2) & Orale & $\mathrm{M}, \mathrm{Mg}$ & 0,03 & 0,67 \\
\hline & & & & & Racine(1) & & $\operatorname{Mac}(1)$ & & $\mathrm{Mm}$ & & 0,33 \\
\hline 40 & Phyllanthus amarus & Euphorbiaceae & Herbe & 2,44 & Tige feuillée & 03 & $\begin{array}{l}\text { Déc, Pil, } \\
\text { Mac }\end{array}$ & Orale & $\mathrm{I}, \mathrm{M}$ & 0,03 & 1 \\
\hline 41 & Ocimum americanum & Lamiaceae & Herbe & 1,63 & Feuille & 02 & Déc & Orale & Mm, I & 0,02 & 1 \\
\hline 42 & Ocimum basilicum & Lamiaceae & Herbe & 0,81 & Feuille & 01 & Cal & Orale & $\mathrm{M}, \mathrm{Mm}$ & 0,01 & 1 \\
\hline 43 & Cassytha filiformis & Lauraceae & Liane & 1,63 & Plante entière & 02 & Pil, Mac & Orale & Gmq, Rs & 0,02 & 1 \\
\hline $\mathbf{N}^{\circ}$ & Noms scientifiques & Familles & TM & CPR & Organes & VUe & Мp & $\mathbf{V a}$ & MT & VI & VUoe \\
\hline 44 & Baphia nitida & Leguminosae & Arbre & 8,13 & Ecorce & 10 & Déc & Orale & $\mathrm{I}, \mathrm{Mm}$ & 0,11 & 1 \\
\hline 45 & $\begin{array}{l}\text { Calliandra } \\
\text { portoricensis }\end{array}$ & Leguminosae & Arbuste & 0,81 & Racine & 01 & Pil & Orale & $\mathrm{M}$ & 0,01 & 1 \\
\hline
\end{tabular}


V. HOUMENOU et al. / Int. J. Biol. Chem. Sci. 11(4): 1851-1871, 2017

\begin{tabular}{|c|c|c|c|c|c|c|c|c|c|c|c|}
\hline 46 & Desmodium velutinum & Leguminosae & Herbe & 1,63 & Feuille & 02 & Trit, Calc & Orale & $\mathrm{I}, \mathrm{Rs}$ & 0,02 & 1 \\
\hline 47 & Dialium guineense & Leguminosae & Arbre & 1,63 & Ecorce & 02 & Déc & Orale & $\mathrm{Mm}, \mathrm{M}$ & 0,02 & 1 \\
\hline 48 & Erythrina senegalense & Leguminosae & Arbre & 0,81 & Feuille & 01 & Déc & Orale & $\mathrm{M}$ & 0,01 & 1 \\
\hline 49 & Parkia biglobosa & Leguminosae & Arbre & 2,44 & $\begin{array}{l}\text { Racine(2), } \\
\text { Ecorce(1) }\end{array}$ & 03 & $\begin{array}{l}\text { Déc (2), } \\
\text { Mac (1) }\end{array}$ & $\begin{array}{l}\text { Orale, } \\
\text { Toilette intime }\end{array}$ & $\begin{array}{l}\text { Rs, M, } \\
\mathrm{Mm}\end{array}$ & 0,03 & $\begin{array}{l}0,67 \\
0,33\end{array}$ \\
\hline 50 & Pterocarpus erinaceus & Leguminosae & Arbre & 4,06 & Ecorce & 05 & Déc & Orale & $\mathrm{Mm}, \mathrm{I}, \mathrm{A}$ & 0,05 & 1 \\
\hline 51 & Schrankia leptocarpa & Leguminosae & Liane & 0,81 & Liane feuillée & 01 & Déc & Orale & I & 0,01 & 1 \\
\hline 52 & Tetrapleura tetraptera & Leguminosae & Arbre & 5,69 & Fruit & 07 & Mac, & $\begin{array}{l}\text { Orale, } \\
\text { Toilette intime }\end{array}$ & M, Rs, I & 0,08 & 1 \\
\hline 53 & Anthocleista vogelii & Loganiaceae & Arbre & 1,63 & Racine & 02 & Déc & Orale & $\mathrm{I}, \mathrm{Mm}$ & 0,02 & 1 \\
\hline 54 & Gossypium hirsutum & Malvaceae & Arbuste & 0,81 & Racine & 01 & Déc & Orale & I & 0,01 & 1 \\
\hline 55 & Sida acuta & Malvaceae & Herbe & 0,81 & Tige feuillée & 01 & Déc & Orale & $\mathrm{Tb}$ & 0,01 & 1 \\
\hline 56 & Khaya senegalensis & Meliaceae & Arbre & 1,63 & Ecorce & 02 & Déc & Orale & $\mathrm{I}, \mathrm{Mm}$ & 0,02 & 1 \\
\hline 57 & $\begin{array}{l}\text { Cissampelos } \\
\text { owariensis }\end{array}$ & Menispermaceae & Liane & 2,44 & Feuille & 03 & $\begin{array}{l}\text { Trit (2) } \\
\text { Déc (1) }\end{array}$ & Orale & $\mathrm{Rs}, \mathrm{Mg}$ & 0,03 & 1 \\
\hline 58 & Ficus sur & Moraceae & Arbre & 0,81 & Fruit & 01 & Pil & Orale & I & 0,01 & 1 \\
\hline 59 & Milicia excelsa & Moraceae & Arbre & 0,81 & Ecorce & 01 & Déc & Orale & $\mathrm{Tb}$ & 0,01 & 1 \\
\hline 60 & Moringa oleifera & Moringaceae & Arbre & 0,81 & Feuille & 01 & Déc & Orale & Urs & 0,01 & 1 \\
\hline 61 & $\begin{array}{l}\text { Musa sapientum var } \\
\text { gounkokwé en goun }\end{array}$ & Musaceae & Herbe & 6,50 & $\begin{array}{l}\text { - Faux tronc (2) } \\
\text { - Fruit (4) } \\
\text { - rhizom(2) }\end{array}$ & 8 & $\begin{array}{l}\text { Déc (2) } \\
\text { Mac (5) } \\
\text { Pil (1) }\end{array}$ & $\begin{array}{l}\text { Orale, } \\
\text { Toilette intime }\end{array}$ & Rs, Ha, M & 0,09 & $\begin{array}{l}0,25 \\
0,50 \\
0,25\end{array}$ \\
\hline 62 & Psidium guajava & Myrtaceae & Arbuste & 0,81 & Feuille & 01 & Déc & Orale & I & 0,01 & 1 \\
\hline 63 & Syzygium aromaticum & Myrtaceae & Arbre & 2,44 & Boutons floraux & 03 & $\begin{array}{l}\text { Déc (2) } \\
\text { Mac (1) }\end{array}$ & Orale & $\mathrm{Mm}, \mathrm{I}$ & 0,03 & 1 \\
\hline 64 & Olax subscorpioidea & Olacaceae & Arbuste & 5,69 & Racine & 07 & $\operatorname{Mac}(6)$ & Orale & Ha, M, I & 0,08 & 1 \\
\hline
\end{tabular}


V. HOUMENOU et al. / Int. J. Biol. Chem. Sci. 11(4): 1851-1871, 2017

\begin{tabular}{|c|c|c|c|c|c|c|c|c|c|c|c|}
\hline & & & & & & & Déc (1) & Toilette intime & & & \\
\hline 65 & Cymbopogon citratus & Poaceae & Herbe & 0,81 & Feuille & 01 & Déc & Orale & Rs & 0,01 & 1 \\
\hline 66 & Pennisetum purpureum & Poaceae & Herbe & 0,81 & Feuille & 01 & Déc & Orale & $\mathrm{Mm}$ & 0,01 & 1 \\
\hline 67 & Zea mays & Poaceae & Herbe & 0,81 & "Barbes"' du fruit & 01 & Cal & Orale & $\mathrm{Ha}$ & 0,01 & 1 \\
\hline 68 & Sesamum indicum & Pedaliaceae & Herbe & 0,81 & Feuille & 01 & Trit & Orale & Rs, M & 0,01 & 1 \\
\hline 69 & Peperomia pellucida & Piperaceae & Herbe & 1,63 & Tige feuillée & 02 & Trit, Mac & Orale & $\mathrm{Ha}, \mathrm{I}, \mathrm{Rs}$ & 0,02 & 1 \\
\hline 70 & Piper guineense & Piperaceae & Liane & 4,88 & Graine & 06 & $\begin{array}{l}\text { Mac (2),Déc } \\
(2), \text { Cal (2) }\end{array}$ & Orale & $\mathrm{I}, \mathrm{M}, \mathrm{Mm}$ & 0,07 & 1 \\
\hline $\mathbf{N}^{\circ}$ & Noms scientifiques & Familles & TM & CPR & Organes & VUe & Мp & $\mathbf{V a}$ & MT & VI & VUoe \\
\hline 71 & Chassalia kolly & Rubiaceae & Arbuste & 1,63 & Racine & 02 & Mac,Déc & Orale & Rs, M & 0,02 & 1 \\
\hline 72 & Hallea stipulosa & Rubiaceae & Arbre & 0,81 & Ecorce & 01 & Déc & Orale & $\mathrm{Gmq}$ & 0,01 & 1 \\
\hline 73 & Morinda lucida & Rubiaceae & Arbre & 4,06 & Racine & 05 & $\begin{array}{l}\text { Pil (3) } \\
\text { Déc (2) }\end{array}$ & Orale & $\begin{array}{l}\mathrm{Mm}, \mathrm{M}, \\
\mathrm{Ha}, \mathrm{I}\end{array}$ & 0,05 & 1 \\
\hline 74 & $\begin{array}{l}\text { Sarcocephalus } \\
\text { latifolius }\end{array}$ & Rubiaceae & Arbuste & 1,63 & Racine & 02 & Déc & Orale & I & 0,02 & 1 \\
\hline 75 & Citrus aurantium & Rutaceae & Arbre & 0,81 & Fruit & 01 & Trit & Orale & M & 0,01 & 1 \\
\hline 76 & $\begin{array}{l}\text { Zanthoxylum } \\
\text { zanthoxyloides }\end{array}$ & Rutaceae & Arbre & 0,81 & Racine & 01 & Déc & Orale & M & 0,01 & 1 \\
\hline 77 & Paullinia pinnata & Sapindaceae & Liane & 0,81 & Racine & 01 & Déc & Orale & $\mathrm{Mm}$ & 0,01 & 1 \\
\hline 78 & Capsicum annuum & Solanaceae & $\begin{array}{l}\text { Sous- } \\
\text { arbrisseau }\end{array}$ & 0,81 & Fruit & 01 & Déc & Orale & I & 0,01 & 1 \\
\hline 79 & Nicotiana tabacum & Solanaceae & Herbe & 2,44 & Feuille & 03 & $\begin{array}{l}\text { Mac (2),Cal } \\
(1)\end{array}$ & Orale & M & 0,03 & 1 \\
\hline 80 & Schwenckia americana & Solanaceae & Herbe & 0,81 & Feuille & 01 & Déc & Orale & I & 0,01 & 1 \\
\hline
\end{tabular}


V. HOUMENOU et al. / Int. J. Biol. Chem. Sci. 11(4): 1851-1871, 2017

\begin{tabular}{|c|c|c|c|c|c|c|c|c|c|c|c|}
\hline 81 & Cola gigantea & Sterculiaceae & Arbre & 0,81 & Cabosse (fruit) & 01 & Cal & Orale & I & 0,01 & 1 \\
\hline 82 & Cola millenii & Sterculiaceae & Arbre & 1,63 & $\begin{array}{l}\text { Feuille(1), } \\
\text { Ecorce(1) }\end{array}$ & 02 & Déc, Mac & Orale & $\mathrm{M}, \mathrm{Tb}$ & 0,02 & $\begin{array}{l}0,5 ; \\
0,5\end{array}$ \\
\hline 83 & Sterculia tragacantha & Sterculiaceae & Arbre & 0,81 & Ecorce & 01 & Mac & Orale & $\mathrm{Tb}$ & 0,01 & 1 \\
\hline 84 & Laportea aestuans & Urticaceae & Herbe & 0,81 & Feuille & 01 & Trit & Voie vaginale & Is & 0,01 & 1 \\
\hline 85 & Vitex doniana & Verbenaceae & Arbre & 0,81 & $\begin{array}{l}\text { Tige feuillée de } \\
\text { son parasite }\end{array}$ & 01 & Déc & Orale & $\mathrm{Mm}, \mathrm{I}$ & 0,01 & 1 \\
\hline 86 & $\begin{array}{l}\text { Hybanthus } \\
\text { enneaspermus }\end{array}$ & Violaceae & Herbe & 0,81 & Feuille & 01 & Pil & Orale & I & 0,01 & 1 \\
\hline 87 & Cissus populnea & Vitaceae & Liane & 0,81 & Racine & 01 & Mac & Orale & Rs & 0,01 & 1 \\
\hline 88 & Aframomum melegueta & Zingiberaceae & Herbe & 7,32 & Fruit, (gousse) & 09 & $\begin{array}{l}\text { Cal (5), Pil } \\
\text { (3), Déc (1) }\end{array}$ & $\begin{array}{l}\text { Orale, Voie } \\
\text { vaginale }\end{array}$ & $\begin{array}{l}\mathrm{Rs}, \mathrm{I}, \mathrm{M}, \\
\mathrm{Ha}, \mathrm{Is}\end{array}$ & 0,1 & 1 \\
\hline 89 & Aframomum sceptrum & Zingiberaceae & Herbe & 0,81 & Feuille & 01 & Déc & Orale & I & 0,01 & 1 \\
\hline 90 & Zingiber officinale & Zingiberaceae & Herbe & 0,81 & Rhizome & 01 & Déc & Orale & I & 0,01 & 1 \\
\hline
\end{tabular}




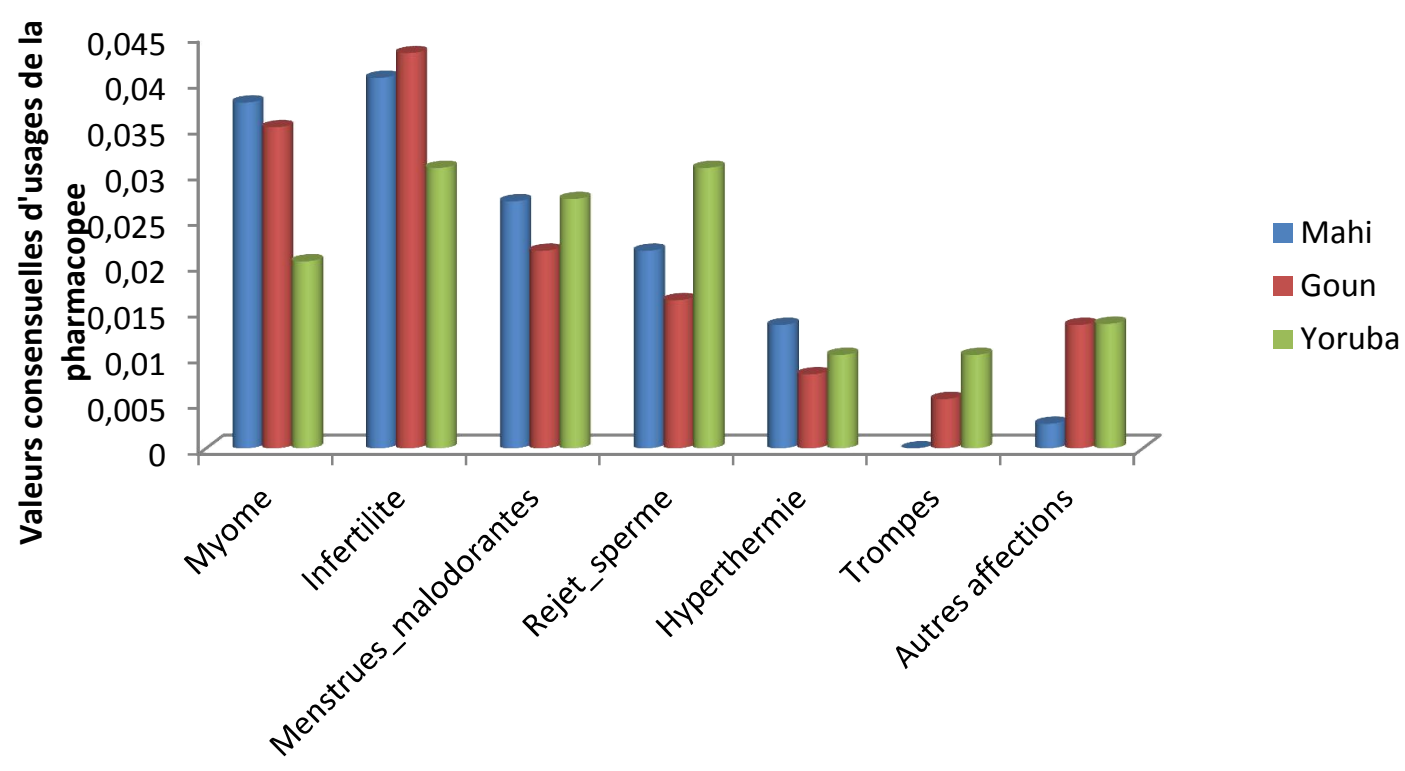

Figure 4 : Histogramme des niveaux de consensus des causes de la stérilité féminine

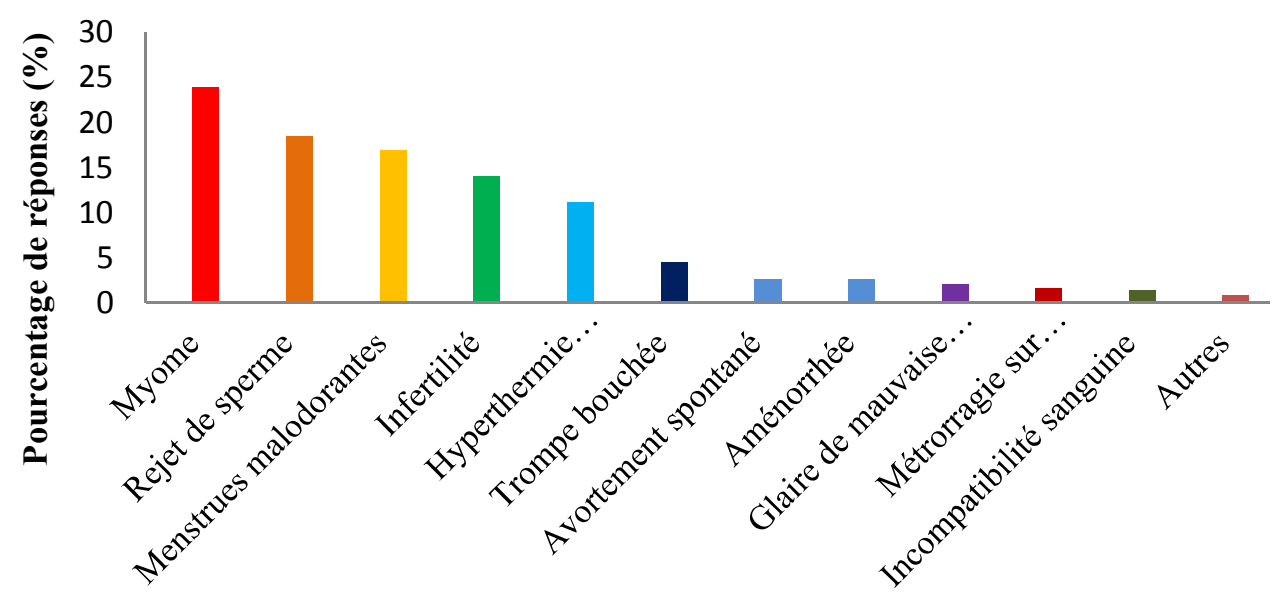

Figure 5 : Diagramme des causes de la stérilité féminine vues en médecine traditionnelle

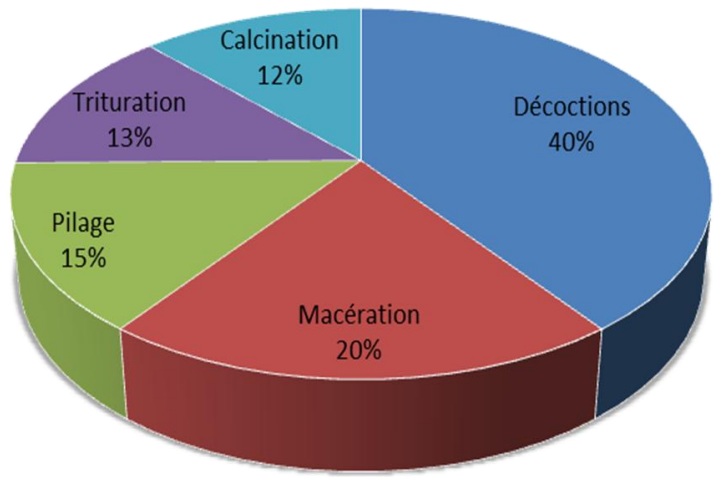

Figure 6 : Diagramme des modes de préparation des recettes

1866 


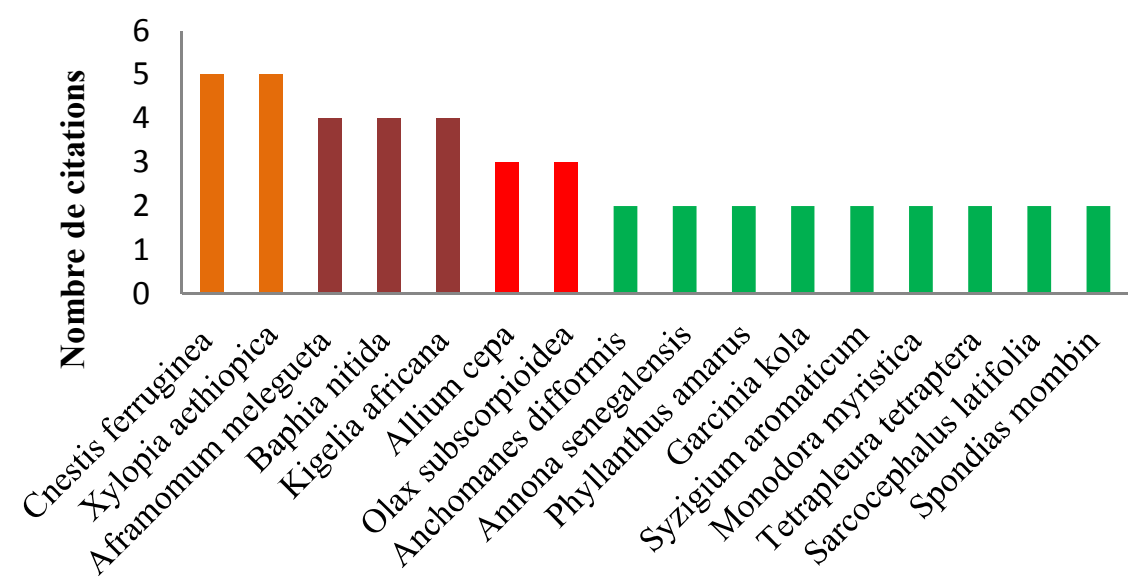

Figure a : plantes utilisées contre l'infertilité

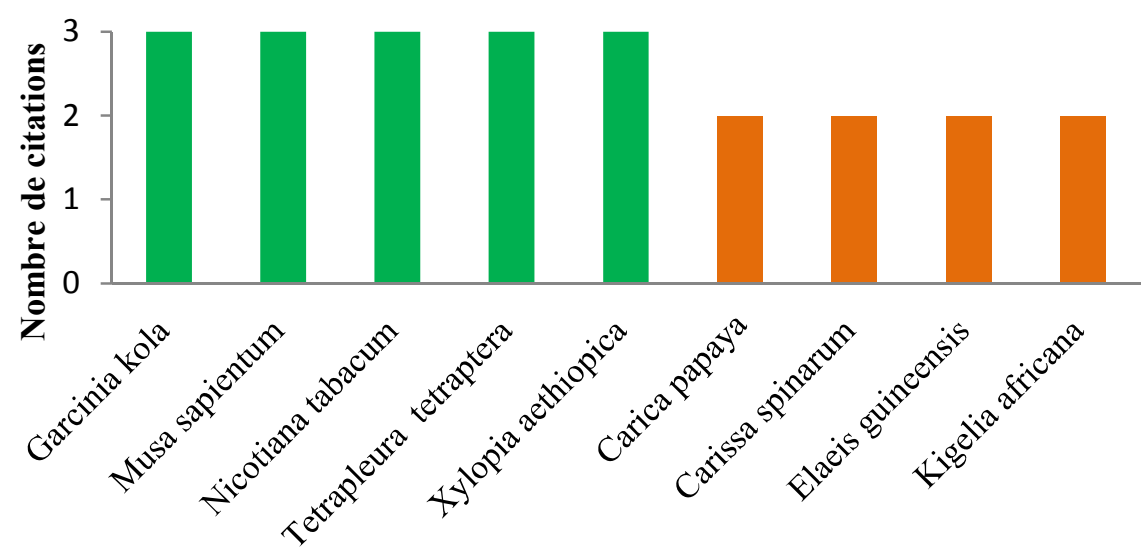

Figure b : Plantes utilisées contre le myome

Cissampelos owariensis

Musa sapientum

Aframomum melegueta

Pupalia lappacea

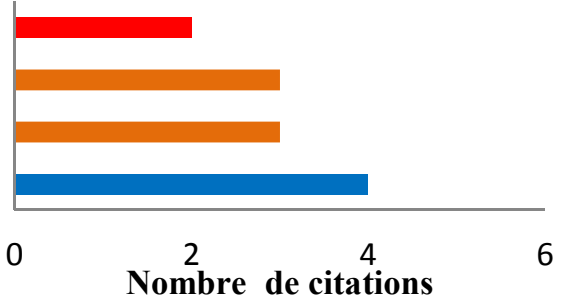

Figure c : Plantes utilisées contre le rejet de sperme

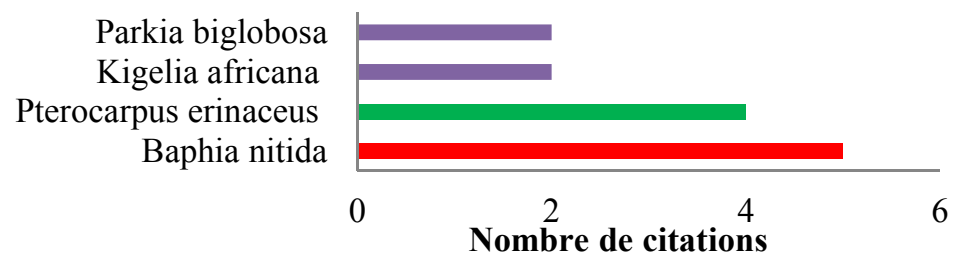

Figure d : Plantes utilisées contre les menstrues malodorantes

Figure 7 : Principales plantes utilisées de façon spécifique dans le traitement de la stérilité féminine. 


\section{DISCUSSION}

Dans cette étude ethnobotanique, les enquêtés, constitués des tradithérapeutes et des personnes ressources sont tous des hommes ; ceci laisse sous-tendre que les femmes sont soumises à leurs maris en matière de gestion des connaissances sur les traitements contre les affections liées à la stérilité féminine. Cependant, Déléké Koko et al. (2011) ont montré que concernant l'exploitation traditionnelle des plantes galactogènes et emménagogues, au sein de tous les groupes socioculturels, les connaissances relatives aux plantes médicinales sont parfaitement partagées entre les hommes et les femmes. Les enquêtes ethnobotaniques ont permis de savoir que les tradithérapeutes et personnes ressources du Bénin ont des connaissances endogènes sur le traitement de la stérilité féminine ; ces faits ont confirmé les travaux de Adomou et al. (2012) qui ont montré que sur 13 affections, la stérilité féminine a été citée en $3^{\text {ème }}$ position comme maladie fréquemment traitée par une vendeuse phytothérapeute. Face à cette difficulté de procréation, plusieurs causes en sont-elles à l'origine. C'est le cas du myome, de l'infertilité, des menstrues malodorantes, $\mathrm{du}$ rejet de sperme, etc. dont le traitement nécessite de nombreuses espèces végétales ( 90 espèces inventoriées) utilisées seules ou en combinaison avec d'autres dans les diverses recettes recensées. Ces résultats sont en accord avec ceux de l'OMS (2001) qui estime que $90 \%$ des recettes recensées en Afrique sont à la base des plantes. Des 90 espèces végétales utilisées dans le traitement de la stérilité féminine, existe une espèce exotique, Syzygium aromaticum qui n'est pas présente dans la flore du Bénin ; ses boutons floraux (clous de girofle) sont importés du Nigéria et vendus dans nos marchés. Les 89 plantes restantes représentent $3,17 \%$ de la flore analytique du Bénin estimée à 2807 espèces (Akoègninou et al., 2006). Cette richesse spécifique est supérieure aux 53 plantes recensées par Nkounkou-Loumpangou et al. (2005) dans les zones Sud et Nord de
Brazzaville au Congo pour le traitement de l'infertilité féminine. Les plantes médicinales intervenant dans le traitement de la stérilité féminine sont diversement utilisées. Certaines recettes utilisent seuls ou en combinaison les organes tels que les feuilles, les racines, les écorces, les fruits, etc. Parmi ces usages, celui des fruits parait sans incidence pour la plante alors que l'usage des feuilles est moins préjudiciable pour la plante que les racines et les écorces. Il est prouvé que l'impact d'une utilisation particulière sur un arbre, dépend de la partie utilisée et de la méthode de prélèvement. La récolte des racines d'un arbre et/ou de son écorce semble avoir plus d'incidences écologiques néfastes que la récolte des feuilles (Cunningham, 2001).

S'agissant des organes utilisés dans les recettes plurispécifiques, il est à remarquer qu'ils n'ont pas la même importance. Certains organes, surtout les fruits et les graines, sont utilisés comme des ingrédients auxilliaires ou adjuvants, permettant de renforcer l'action thérapeutique des composantes principales des recettes et de traiter les symptômes secondaires de la maladie (Joy et al., 2001); c'est le cas des fruits ou graines de Tetrapleura tetraptera, Aframomum melegueta, Monodora myristica, Garcinia kola, Piper guineense, etc.

Concernant les modes de confection des médicaments, la décoction et la macération sont les plus citées; ce qui corrobore les travaux de NkounkouLoumpangou et al. (2005) mais différents de ceux de Béné et al. (2016) qui ont montré que la décoction et le broyage sont les plus importants modes de préparation en pharmacopée traditionnelle. Certaines espèces végétales ont des spécificités d'action contre une cause donnée. C'est ainsi que Cnestis ferruginea, Kigelia africana et dans une moindre mesure Olax subscorpioidea sont les plus citées contre l'infertilité ; Garcinia kola, Nicotiana tabacum, Tetrapleura tetraptera sont les plus énumérés contre le myome; Pupalia lappacea est la plus sollicitée contre le rejet de sperme et Pterocarpus erinaceus 
l'est contre les menstrues malodorantes. La spécificité d'une plante pour le traitement d'une affection donnée vient appuyer les résultats des travaux de Déléké Koko et al. (2009) qui ont affirmé que sur 57 plantes emménagogues et galactogènes recensées sur les terroirs autour de la Zone Cynégétique de la Pendjari, les plus utilisées sont Vitellaria paradoxa, Sarcocephalus latifolius, Daniellia oliveri, Adansonia digitata, Terminalia avicennioides, Sorghum bicolor et Ficus gnaphalocarpa.

De nombreuses plantes citées par nos tradithérapeutes et personnes ressources du Sud-Bénin pour le traitement de l'une ou l'autre des causes de la stérilité féminine sont reconnues également comme des plantes médicinales utilisées dans le traitement de l'infertilité féminine à Brazzaville au Congo (Nkoukou-Loumpangou et al., 2005). C'est le cas de Cnestis ferruginea, Sarcocephallus latifolius, Psidium guajava, Bridelia ferruginea, Schwenckia americana, Sida acuta, Anchomanes difformis, Morinda lucida. Milicia excelsa et Cassytha filiformis qui sont respectivement utilisées pour déboucher les trompes et traiter la glaire cervicale comme au Sud-Bénin.

Parmi les recettes médicamenteuses recensées, il y a presque autant de recettes monospécifiques que de recettes plurispécifiques alors qu'il est plus souhaitable que la proportion de recettes plurispécifiques soit la plus faible possible comme dans le cas des travaux de Zerbo et al. (2007) et de Béné (2016) qui affirment que la prépondérance des recettes monospécifiques est à l'avantage des patients et que les associations des plantes mal assorties sont parfois dangereuses.

La contribution des plantes à la constitution des recettes (CPR) est relativement plus élevée chez certaines plantes que chez d'autres. C'est le cas des espèces comme Aframomum melegueta, Baphia nitida, Garcinia kola, Kigelia africana, Musa sapientum, Olax subscorpioidea, Pupalia lappacea,
Tetrapleura tetraptera et Xylopia aethiopica qui possèdent un CPR plus élevé et par conséquent ont une valeur d'importance relativement supérieure à celle des autres espèces.

\section{Conclusion}

Dans la zone d'étude, la pharmacopée traditionnelle utilise 90 espèces végétales appartenant à quatre types morphologiques, 86 genres et 47 familles pour traiter la stérilité féminine sous ses différentes formes (myome, infertilité, menstrues malodorantes, rejet de sperme, hyperthermie abdominale, etc.). 123 recettes impliquant feuilles, racines, fruits, écorces, graines, tubercules, plantes entières, organes floraux et faux tronc et cinq principaux modes de préparation (décoctions, macérations, pilage, calcination, trituration) sont recensés. Plantes et recettes ont des spécificités d'action et sont plus indiquées dans le traitement d'une forme particulière de cause de stérilité. La quasi-totalité des enquêtés dans cette étude, étant des hommes, il est important de l'étendre aux femmes vendeuses de plantes médicinales dans nos marchés. Le mode d'utilisation étant essentiellement oral, le criblage phytochimique au laboratoire est nécessaire pour connaître les composés chimiques contenus dans les principales plantes utilisées et leur degré de toxicité.

\section{CONFLIT D'INTERETS}

Les auteurs déclarent qu'il n'existe aucun conflit d'intérêts relatif à cet article.

\section{CONTRIBUTIONS DES AUTEURS}

$\mathrm{VH}$ a rédigé le protocole de recherche et a conduit la collecte des données, le dépouillement, le traitement statistique des données, la rédaction et la soumission du manuscrit; AA a lu et a contribué à la correction et à la réalisation des tableaux et figures; MGT et HY ont lu et ont contribué à la correction et à l'identification des plantes. AD, JG et AA ont lu et ont corrigé. 


\section{REMERCIEMENTS}

Les auteurs remercient Monsieur Michel GBAGUIDI pour l'analyse statistique des données. Ils expriment leur reconnaissance à l'égard des tradithérapeutes et personnes ressources pour leur disponibilité.

\section{REFERENCES}

Adjanohoun EJ, Adjakidjè V, Ahyi MRA, Aké Assi L, Akoègninou A, d'Almeida J, Apovo F, Boukef K, Chadare M, Cusset G, Dramane K, Eyme J, Gassita JN, Gbaguidi N, Goudoté E, Guinko S, Hougnon P, Issa LO, Kéita A, Kiniffo HV, Koné-Bamba D, Musampa Nseyya A, Saadou M, Sodogandji Th, de Souza S, Tchabi A, Zinsou Dossa C, Zohoun Th. 1989. Médecine traditionnelle et pharmacopée, Contribution aux études ethnobotaniques et floristiques en République Populaire $d u$ Benin $\left(2^{\mathrm{e}}\right.$ édition). ACCT : Paris.

Adomou AC, Akoègninou A, Sinsin B, de Foucaul B, van der Maesen LJG. 2007. Biogeographical analysis of the vegetation in Benin. Acta Botanica Gallica, 154: 221233.

DOI: 10.1080/12538078.2007.10516053.

Adomou AC, Yedomonhan H, Djossa B, Legba SI, Oumorou M, Akoegninou A. 2012. Etude ethnobotanique des plantes médicinales vendues dans le marché d'Abomey- Calavi au Bénin. Int. J. Biol. Chem. Sci., 6(2): 745-772. DOI: http://ajol.info/index.php/ijbcs

Akoègninou A. 2004. Recherches botaniques et écologiques sur les forêts actuelles du Benin. Thèse d'état, Université de Cocody-Abidjan, Côte d'Ivoire, p 326.

Akoègninou $\mathrm{A}$, van der Burg $\mathrm{WJ}$, van der Maesen LJG, Adjakidjè V, Essou JP, Sinsin B, Yédomonhan H. 2006. Flore analytique $d u$ Benin. Backhuys Publishers : Leiden, The Netherlands.

Assogbadjo AE, Glèlè Kakaï R, Chadare FJ, Thomson L, Kyndt T, Sinsin B, Van Damme P. 2008. Folk classification, perception, and preferences of baobab products in West Africa: Consequences for species conservation and improvement. Econ Bot, 62: 74-84.

Akare Biyoghe B. 2010. Conceptions et comportements des Fang face aux questions de fécondité et de stérilité, regard anthropologique sur une société patrilinéaire du Gabon. Thèse de doctorat en médecine, Université de Metz, France, p. 353 .

Béné K, Camara D, Fofie NBY, Kanga Y, Yapi AB, Yapo YC, Ambe SA, Zirihi GN. 2016. Etude ethnobotanique des plantes médicales utilisées dans le département de Transua, District du Zanzan (Côte d'Ivoire). Journal of Animal \& Plant Sciences, 27(2): 4230-4250. DOI: http://www.m.elewa.org/JAPS

Betti JL, Lejoly J. 2000. Les plantes indiquées comme anthelminthiques en thérapie traditionnelle dans la réserve de biosphère du Dja (Cameroun). Soma, 1: 4-16.

Byg A, Baslev H. 2011. Diversity and use of palms in Zahamena, eastern Madagascar. Biodiversity and Conservation, 10: 951970.

CTA. 2007. Les Plantes Médicinales. Wageningen, Pays-Bas. 35 p. Site Web: www.cta.int.

Cunningham AB. 2001. Applied Ethnobotany. People, Wild Plant Use and Conservation. People and Plants. Conservation Manuel. Earthscan, 300p.

Dansi A, Adjatin A, Adoukonou-Sagbadja H, Faladé V, Yedomonhan H, Odou D, Dossou B. 2008. Traditional leafy vegetables and their use in the Benin Republic. Genet. Resour. Crop Evol., 55: 1239-1256. DOI 10.1007/s10722-0089324-z

Dassou GH, Ogni CA, Yédomonhan $H$, Adomou AC, Tossou M, Dougnon JT, Akoègninou A. 2014. Diversité, ethnobotanique et vulnérabilité des plantes à usages vétérinaires au Nord-Bénin. Int. J. Biol. Chem. Sci., 8(1): 189-210. DOI: http://ajol.info/index.php/ijbcs 
Dassou GH, Adomou AC, Yédomonhan H, Ogni AC, Tossou GM, Dougnon JT, Akoègninou A. 2015. Flore médicinale utilisée dans le traitement des maladies et symptômes animaux au Bénin. Journal of Animal \& Plant Sciences, 26(1): 40364057.DOI: http://www.m.elewa.org/JAPS.

Delvaux C, Sinsin B. 2002. Les plantes médicinales dans la forêt classée des Monts Kouffé au centre Bénin : Stratégie de conservation, de restauration et de production compatible avec le développement local. SOMA, 1 : 73-81.

Déléké Koko IKE, Djego J, Gbénou J, Hounzangbe-Adoté SM, Brice Sinsin 2009. Etude ethnobotanique des plantes galactogènes et emménagogues utilisées dans les terroirs riverains à la Zone Cynégétique de la Pendjari. Int. J. Biol. Chem. Sci., 3(6): 1226-1237. URL: http://ajol.info/index.php/ijbcs

Déléké Koko IKE. 2011. Exploitation traditionnelle des plantes galactogènes et emménagogues: ethnobotanique, efficacité thérapeutique, valeur d'usage et statut de conservation des principales plantes utilisées dans la Réserve de la Biosphère de la Pendjari (Bénin). Thèse de doctorat unique, Université d'Abomey-Calavi, Bénin, p. 275.

Dossou ME, Houessou GL, Lougbégnon OT, Tenté AHB, Codjia JTC. 2012. Étude ethnobotanique des ressources forestières ligneuses de la forêt marécageuse d'Agonvè et terroirs connexes au Bénin. Tropicultura, 30(1): 41-48.

Joy PP, Thomas J, Mathew S, Skaria BP. 2001. Medicinal Plants. Tropical, Horticulture, 2: 449-632.

Keirungi J, Fabricius C. 2005. Selecting medicinal plants for cultivation at Nqabara on the Eastern Cape Wild Coast, South Africa. South African Journal of Sciences, 101: 497-501.

Lahsissene H, Kahouadji A, Tijane M, Hseini S. 2009. Catalogue des plantes médicinales utilisées dans la région de zaër, Maroc occidental. Lejeunia, Revue de Botanique, 186 : 1-30. URL: http://popups.ulg.ac.be/0457-4184.

Nkounkou-Loumpangou C, BinimbiMassengo A, Nzonzi J, Ouamba JM, Abena AA, Diatewa M. 2005. Inventaire des plantes médicinales utilisées dans le traitement de l'infertilité féminine à Brazzaville. Phytothérapie, 6: 252-259.

OMS. 2001. Rapport final de la première réunion du comité régional d'experts de l'OMS sur la médecine traditionnelle. Harare, Zimbabwe 23 Nov. 2001. 41p.

RGPH4. 2013. Effectifs de la population des villages et quartiers de ville du Bénin. INSAE. Février 2016. 85p.

Saadou M. 2008. Proposition de méthode d'étude ethnobotanique: cas du Niger. Atelier international sur l'harmonisation des méthodes d'étude de la flore et de la végétation en Afrique de l'Ouest tenu du 4 au 9 août 2008 .

Zerbo P, Millogo-Rasolodimby J, NacoulmaOuedraogo OG, Van Damme P. 2007. Contribution à la connaissance des plantes médicinales utilisées dans les soins infantiles en pays San, au Burkina Faso. Int. J. Biol. Chem. Sci., 1(3): 262-274. URL: http://ajol.info/index.php/ijbcs 